Alicja Jokiel-Rokita and Rafae Topolnicki (Wrocław)

\title{
ESTIMATION OF THE RATIO OF A GEOMETRIC PROCESS
}

Abstract. We propose some estimators of the ratio parameter of a geometric process, i.e. of a stochastic process $\left\{X_{i}, i=1,2, \ldots\right\}$ for which there exists a positive real number $a$, called the ratio parameter, such that $\left\{Y_{i}=a^{i-1} X_{i}, i=1,2, \ldots\right\}$ forms a renewal process. We assume that the cumulative distribution function $F$ of the random variables $Y_{i}, i=1,2, \ldots$, is completely unknown. We compare the accuracy of the proposed estimators of the ratio with known estimators given by Lam (1992) and by Chan et al. (2006), and also with the maximum likelihood estimators derived under the assumption that $F$ has a known form.

1. Introduction. A stochastic process $\left\{X_{i}, i=1,2, \ldots\right\}$ is a geometric process (GP) if there exists a positive real number $a$ such that $\left\{Y_{i}=\right.$ $\left.a^{i-1} X_{i}, i=1,2, \ldots\right\}$ forms a renewal process. This notion was introduced by Lam [L1] and thoroughly investigated in [L4]. The number $a$ is called the ratio of the GP. Clearly, a GP is stochastically increasing if $0<a \leq 1$, and stochastically decreasing if $a \geq 1$. A GP is a renewal process if $a=1$. Thus, a GP is a simple monotone process and is a generalization of a renewal process.

A GP process under the reparametrization $a=d^{-1}$ is called a quasirenewal process (see for example [WP]).

Many important theoretical properties of GP's have been investigated by Lam [L2] (see also [BLZ]). The GP model leads to tractable solutions for some reliability problems ([L2] and [LZ]).

2010 Mathematics Subject Classification: Primary 62F10; Secondary 62G05.

Key words and phrases: geometric process, least squares estimation, weighted least squares estimation, second order least squares estimation, minimum contrast estimation.

Received 10 August 2016.

Published online 3 March 2017. 
Denote $\mu=E\left(X_{1}\right)$ and $\sigma^{2}=\operatorname{Var}\left(X_{1}\right)$. Then

$$
E\left(X_{i}\right)=\frac{\mu}{a^{i-1}} \quad \text { and } \quad \operatorname{Var}\left(X_{i}\right)=\frac{\sigma^{2}}{a^{2(i-1)}} .
$$

Thus $a, \mu$ and $\sigma^{2}$ are tree parameters which completely determine the mean and the variance of $X_{i}$. The mean parameter $\mu$ indicates the initial level of a trend, and the ratio parameter $a$, which is the ratio of the expected present outcome to the previous outcome, reveals the progression of the trend while eliminating random noises. The ratio $a$ is important for example in epidemic modeling as it is related to the basic reproductive rate $R_{0}$ which is the number of new infections created per each infected person (see [CYLH]). When $R_{0}>1$, the epidemic will attack more and more people in each round of infection so that the expected number of daily incidences, $E\left(X_{i}\right)=\mu / a^{i-1}$, will be increasing over time $i$, implying that $a<1$. On the other hand, when $R_{0}<1$, the epidemic reduces its force of infection in each round and stops eventually, so that $E\left(X_{i}\right)$ with $a>1$ will be decreasing over time $i$. Because of its relationship with $R_{0}$, the ratio $a$ provides an important indicator of whether an epidemic is under control.

The GP model is also an attractive candidate for reliability problems involving nonstationary processes. A GP process can be used e.g. to model hardware maintenance processes, software reliability growth processes in the testing or operation phase, hardware repair times, and hardware reliability growth in burn-in stage. More extensions or applications of the GP model can be found for example in [F], [S], [L4], [C], [YTF] and [YTLC].

Lam [L3] described in detail tests that check whether the data are consistent with a GP and whether $a=1$. He also proposed nonparametric estimators of $a, \mu$ and $\sigma^{2}$ (i.e. under the assumption that the distribution function $F$ is completely unknown), which we recall in Section 2.1.

Parametric inference for GP's was considered by Lam and Chan [LC], Chan et al. [CLL, Aydoğdu et al. [ASK] and Kamal et al. [KZSA]. Lam and Chan [LC] considered statistical inference for a GP with lognormal distribution for the first occurrence time $X_{1}$ of an event. Under the assumption that $X_{1}$ follows the gamma distribution, the statistical inference problem for the GP was studied by Chan et al. [CLL, where the unknown model parameters were estimated by the maximum likelihood method and by the modified moment method, previously proposed by Lam [L3]. Aydoğdu et al. ASK] derived explicit estimators of the parameters in a GP when $X_{1}$ is assumed to be Weibull using the method of modified maximum likelihood proposed by Tiku [T]. Kamel et al. [KZSA] used the Weibull geometric process model to analyze accelerated life testing under constant stress and derived the maximum likelihood estimates of the model parameters and their confidence intervals using both asymptotic and parametric bootstrap methods. 
In many papers, the optimal repair-replacement problem, under various assumptions, is studied for a repairable system, modeled by a GP (see e.g. [L1], [S], and recently [WZ]).

In the present paper we take up the problem of point estimation of the ratio parameter $a$ in the case when the cumulative distribution function $F$ of the random variables $Y_{i}, i=1,2, \ldots$, is completely unknown. The article is organized as follows. In Section 2 we present some methods of estimation based on $\ln X_{1}, \ldots, \ln X_{n}$. In the GP considered, $\ln X_{i}=\ln Y_{i}-(i-1) \ln a$, and to estimate the parameter $a$ we apply some regression techniques. We recall the estimator based on the least squares method (Section 2.1), derived by Lam [L3], and we propose some new estimators: based on the second order least squares method (Section 2.2), and based on quantile regression (Section 2.3). In Section 3 we recall the ordinary least squares estimator derived by Chan et al. [CYLH], and propose some new estimators: based on the weighted least squares method, and based on a profile weighted least squares method. In Section 4 we present maximum likelihood (ML) estimators for some GP models which will be used in a simulation study. The accuracy of the estimators of the ratio parameter $a$ under study is examined in Section 5 for simulated GP models. Moreover, findings concerning the robustness of the ML estimators are presented. In Section 6 the results are applied to some real data. Section 7 contains concluding remarks and some prospects.

2. Estimators of the ratio parameter based on logarithms of observations. Notice that since $Y_{i}=a^{i-1} X_{i}, i=1, \ldots, n$, are i.i.d. random variables, we can write

$$
\ln X_{i}=\lambda+(1-i) \ln a+e_{i}, \quad i=1, \ldots, n,
$$

where

$$
\lambda:=E\left(\ln Y_{i}\right), \quad e_{i}:=\ln Y_{i}-\lambda,
$$

and $e_{i}, i=1, \ldots, n$, are i.i.d random variables with $E\left(e_{i}\right)=0$ and

$$
\operatorname{Var}\left(e_{i}\right)=\operatorname{Var}\left(\ln Y_{i}\right)=: \tau^{2} .
$$

Denote $\vartheta=\ln a$. Equation (2.1) takes the form

$$
\ln X_{i}=\lambda+(1-i) \vartheta+e_{i}, \quad i=1, \ldots, n,
$$

and defines a linear regression model with dependent variables $\ln X_{i}$ and nonrandom regressors $1-i, i=1, \ldots, n$. To determine estimators of the parameter $a$ we can use methods of estimation of the coefficient $\vartheta$ of linear regression given by 2.2), and take $\hat{a}=\exp (\hat{\vartheta})$.

2.1. The LS method. When the distribution function $F$ is completely unknown, Lam [L3] has proposed the following method of estimation of the 
ratio parameter. Denote

$$
Q_{n}^{\mathrm{LS}}(\vartheta, \lambda)=\sum_{i=1}^{n}\left[\ln X_{i}-(\lambda+(1-i) \vartheta)\right]^{2} .
$$

The so-called LS estimators of $\vartheta$ and $\lambda$ minimize $(2.3)$, and are given by

$$
\begin{aligned}
& \hat{\vartheta}_{\mathrm{LS}}=\frac{6}{n(n-1)(n+1)} \sum_{i=1}^{n}(n-2 i+1) \ln X_{i}, \\
& \hat{\lambda}_{\mathrm{LS}}=\frac{2}{n(n+1)} \sum_{i=1}^{n}(2 n-3 i+1) \ln X_{i} .
\end{aligned}
$$

The LS estimator $\hat{\tau}_{\mathrm{LS}}^{2}:=\frac{1}{n-2} Q_{n}^{\mathrm{LS}}(\hat{\vartheta}, \hat{\lambda})$ of the parameter $\tau^{2}$ is given by

$$
\begin{aligned}
\hat{\tau}_{L S}^{2} & =1 \\
& \frac{1}{n-2}\left[\sum_{i=1}^{n} \ln ^{2} X_{i}-\frac{1}{n}\left(\sum_{i=1}^{n} \ln X_{i}\right)^{2}-\frac{\hat{\vartheta}_{\mathrm{LS}}}{2} \sum_{i=1}^{n}(n-2 i+1) \ln X_{i}\right] .
\end{aligned}
$$

Denote $\hat{a}_{\mathrm{LS}}=\exp \left(\hat{\vartheta}_{\mathrm{LS}}\right)$ and let $\hat{a}_{\mathrm{LS}(n)}$ be the LS estimator of the parameter $a$ based on the $n$ observations $\ln X_{1}, \ldots, \ln X_{n}$.

The following theorem, proved in [LZCL], gives the asymptotic distribution of the estimator $\hat{a}_{L S(n)}$.

TheOREM 2.1. If $\tau^{2}<\infty$, then

$$
n^{3 / 2}\left(\hat{a}_{\mathrm{LS}(n)}-a\right) \stackrel{D}{\longrightarrow} \mathcal{N}\left(0,12 a^{2} \tau^{2}\right) \quad \text { as } n \rightarrow \infty .
$$

REMARK 2.2. The estimators $\hat{\vartheta}_{\mathrm{LS}}$ and $\hat{\lambda}_{\mathrm{LS}}$ given by 2.4 and 2.5), respectively, are the maximum likelihood (ML) estimators of $\vartheta$ and $\lambda$ in the case when $\ln Y_{i}, i=1, \ldots, n$, are normally distributed, i.e. when $F$ is the cumulative distribution function of a lognormal distribution.

REMARK 2.3. The estimators $\hat{\vartheta}_{\text {LS }}$ and $\hat{\lambda}_{\text {LS }}$ are unbiased estimators of $\vartheta$ and $\lambda$, respectively, and they are linear functions of the logarithms of the observations. Moreover, by the Gauss-Markov theorem, they are uniformly not worse than any estimators $\tilde{\vartheta}$ and $\tilde{\lambda}$ from the class of unbiased estimators which are linear functions of the logarithms of the observations, with respect to the mean square error. From the above properties of $\hat{\vartheta}_{\mathrm{LS}}$ we see that $\hat{a}_{\mathrm{LS}}$ is a biased estimator of $a$, and we know nothing about its mean square error in comparison with other estimators.

2.2. The SLS method. The second order least squares (SLS) method of estimation was proposed by Wang [W1], [W2] in nonlinear measurement error models. This method, which is based on the first two conditional moments of the response variable given the observed predictor variables, extends the ordinary least squares (OLS) estimation by including in the criterion 
function the distance of the squared response variable to its second conditional moment. Wang showed that under some regularity conditions the SLS estimator is consistent and asymptotically normally distributed. Furthermore, Wang and Leblanc [WL] compared the SLS estimator with the OLS estimator in general nonlinear models. They showed that the SLS estimator is asymptotically more efficient than the OLS estimator when the third moment of the random error is nonzero.

We apply the SLS method in our estimation problem. Under the observations $\ln X_{i}, i=1, \ldots, n$, the SLS estimators $\hat{a}_{\mathrm{SLS}}, \hat{\lambda}_{\mathrm{SLS}}$ and $\hat{\tau}_{\mathrm{SLS}}^{2}$ of the parameters $a, \lambda$ and $\tau^{2}$ respectively have to minimize the function

$$
Q_{n}^{\mathrm{SLS}}\left(a, \lambda, \tau^{2}\right)=\sum_{i=1}^{n} \rho_{i}^{T}\left(a, \lambda, \tau^{2}\right) W_{i} \rho_{i}\left(a, \lambda, \tau^{2}\right),
$$

where

$$
\rho_{i}\left(a, \lambda, \tau^{2}\right)=\left(\ln X_{i}-g_{i}(a, \lambda), \ln ^{2} X_{i}-g_{i}^{2}(a, \lambda)-\tau^{2}\right)^{T}
$$

with

$$
g_{i}(a, \lambda)=\lambda+(1-i) \ln a,
$$

and $W_{i}$ is a $2 \times 2$ nonnegative definite matrix which may depend on $i$. In the special case when $W_{i}=I, i=1, \ldots, n$, minimizing $Q_{n}^{\mathrm{SLS}}$ with respect to $a$, $\lambda$ and $\tau^{2}$ is equivalent to minimizing

$T\left(\vartheta, \lambda, \tau^{2}\right):=\sum_{i=1}^{n}\left[\ln X_{i}-\lambda-(1-i) \vartheta\right]^{2}+\sum_{i=1}^{n}\left[\ln ^{2} X_{i}-(\lambda+(1-i) \vartheta)^{2}-\tau^{2}\right]^{2}$

with respect to $\vartheta, \lambda$ and $\tau^{2}$. The solution of the above optimization problem is

$$
\begin{aligned}
& \hat{\vartheta}_{T}=\frac{6 \sum_{i=1}^{n}\left(\frac{n+1}{2}-i\right) \ln ^{2} X_{i}}{(n-1)(n+1) \sum_{i=1}^{n} \ln X_{i}}, \\
& \hat{\lambda}_{T}=\frac{1}{n} \sum_{i=1}^{n} \ln X_{i}+\frac{n-1}{2} \hat{\vartheta} \\
& \hat{\tau}_{T}^{2}=\frac{1}{n} \sum_{i=1}^{n}\left[\ln ^{2} X_{i}-(\hat{\lambda}+(1-i) \hat{\vartheta})^{2}\right]
\end{aligned}
$$

and we take

$$
\hat{a}_{T}=\exp \left(\hat{\vartheta}_{T}\right) .
$$

Wang and Leblanc [WL, Corollary 1] have derived optimal weighting matrices, say $U_{i}$, for which the SLS estimators of the parameters of the general regression model with random regressors are asymptotically the most efficient (under some assumptions). The optimal weighting matrices $U_{i}$, 
$i=1, \ldots, n$, are such that

$$
U_{i}^{-1}=\frac{1}{\tau^{2}\left(\mu_{4}-\tau^{4}\right)-\mu_{3}^{2}}\left(\begin{array}{cc}
u_{i 1} & u_{i 2} \\
u_{i 3} & \tau^{2}
\end{array}\right)
$$

where

$$
\begin{aligned}
& u_{i 1}=\mu_{4}+4 \mu_{3} g_{i}(a, \lambda)+4 \tau^{2} g_{i}^{2}(a, \lambda)-\tau^{4}, \\
& u_{i 2}=-\mu^{3}-2 \tau^{2} g_{i}(a, \lambda), \\
& u_{i 3}=-\mu^{3}-2 \tau^{2} g_{i}(a, \lambda),
\end{aligned}
$$

$g_{i}(a, \lambda), i=1, \ldots, n$, are given by 2.8$)$, and $\mu_{3}=E\left(e^{3}\right), \mu_{4}=E\left(e^{4}\right)$ denote the third and fourth moments of the random error, respectively. The optimal weighting matrix $U_{i}$ involves the unknown parameters $a, \lambda$ and $\tau$. Although in the model considered the regressors are nonrandom, we propose to derive the SLS estimators of the parameters $a, \lambda$ and $\tau$ which minimize $Q_{n}^{\text {SLS }}$ with $W_{i}=\hat{U}_{i}$, where $\hat{U}_{i}$ are estimators of $U_{i}, i=1, \ldots, n$, obtained in the following two-stage procedure. First, the LS estimators $\hat{a}_{\mathrm{LS}}, \hat{\lambda}_{\mathrm{LS}}$ and $\hat{\tau}_{\mathrm{LS}}^{2}$ of unknown parameters are computed according to $2.4-2.6$. Then, we estimate the elements of $U_{i}$ using the corresponding moments from the residuals. Finally, we minimize the objective function 2.7) with $W_{i}=\hat{U}_{i}$, to obtain the second stage estimators. The procedure could be iterated further, but we found that the two-stage procedure is usually adequate and yields much more reliable estimates of $a$ than 2.12). This two-stage procedure differs from the approach presented by Wang and Leblanc [WL where in the first step the identity weights $W_{i}=I, i=1, \ldots, n$, were employed, yielding first-stage estimators given by $2.9-2.11$. We decided to use the LS method in the first step, which corresponds to the weights $W_{i}=\left(\begin{array}{ll}1 & 0 \\ 0 & 0\end{array}\right)$, due to its scale invariance. We denote by $\hat{a}_{\text {SLS }}$ the value minimizing (2.7) with $W_{i}=\hat{U}_{i}$.

REMARK 2.4. Let $Z_{i}=c X_{i}$, for an arbitrary positive constant $c$, be a transformed random variable $X_{i}, i=1, \ldots, n$. Then the ratio parameter of the geometric process $\left\{Z_{i}, i=1,2, \ldots\right\}$ is the same as the ratio parameter $a$ of the target process $\left\{X_{i}, i=1,2, \ldots\right\}$. Therefore, we expect that the change of scale should not affect the estimate of $a$. Unfortunately, applying the SLS method with constant matrices $W_{i}, i=1, \ldots, n$, to the logarithms of the observations $X_{i}, \ldots, X_{n}$ gives a different estimate of $a$ than when applying it to the logarithms of $Z_{i}, \ldots, Z_{n}$. If the matrices $W_{i}$ depend on data, as in the case when $W_{i}=\hat{U}_{i}$, the differences are much smaller.

2.3. The quantile regression method. In this section, to estimate the unknown parameter $a$ we use the quantile regression method (see for example [KB] ) instead of the classical linear regression. One advantage of 
quantile regression, relative to the ordinary least squares regression, is that quantile regression estimates are more robust against outliers in response measurements.

Consider the family $\left\{Q_{n}^{q p}: p \in(0,1)\right\}$ of objective functions

$$
Q_{n}^{q p}\left(\vartheta, \lambda_{p}\right)=\sum_{i=1}^{n} r_{i}\left(\ln X_{i}, \lambda_{p}, \vartheta\right),
$$

where

$$
\begin{aligned}
r_{i}\left(\ln X_{i}, \lambda_{p}, \vartheta\right) \\
\quad= \begin{cases}p\left[\ln X_{i}-\left(\lambda_{p}+(1-i) \vartheta\right)\right] & \text { if } \ln X_{i}-\left(\lambda_{p}+(1-i) \vartheta\right) \geq 0 \\
(1-p)\left[\lambda_{p}+(1-i) \vartheta-\ln X_{i}\right] & \text { if } \ln X_{i}-\left(\lambda_{p}+(1-i) \vartheta\right)<0\end{cases}
\end{aligned}
$$

and

$$
\lambda_{p}=q_{p}\left(\ln Y_{i}\right)
$$

denotes the $p$-quantile of the distribution of the random variable $\ln Y_{i}, i=$ $1, \ldots, n$. The so-called $q p$ estimates $\hat{\vartheta}_{p}$ and $\hat{\lambda}_{p}$ of $\vartheta$ and $\lambda_{p}$, respectively, minimize 2.14. We denote $\hat{a}_{p}=\exp \left(\hat{\vartheta}_{p}\right)$.

A special case of a quantile regression estimator is the median regression estimator which minimizes the following sum of absolute errors:

$$
Q_{n}^{\operatorname{med}}\left(\vartheta, \lambda_{p}\right)=\sum_{i=1}^{n}\left|\ln X_{i}-\left(\lambda_{p}+(1-i) \vartheta\right)\right| .
$$

REMARK 2.5. In the model considered, theoretical quantile regression lines are parallel, therefore we can expect that for example $\hat{\vartheta}_{1 / 2} \approx \hat{\vartheta}_{1 / 3}$.

Based on an extensive simulation study, we have decided to focus on the so-called trimean estimator, defined as

$$
\hat{a}_{\mathrm{TM}}=\left(\hat{a}_{1 / 4}+2 \hat{a}_{1 / 2}+\hat{a}_{3 / 4}\right) / 4 \text {. }
$$

\section{Estimators of the ratio parameter based directly on observa- tions}

3.1. The OLS method. In CYLH the ordinary least squares estimation method, based on the observations $X_{1}, \ldots, X_{n}$, was applied to obtain estimators of the GP model parameters $a$ and $\mu$. The so-called ordinary least squares (OLS) estimators $\hat{a}_{\mathrm{OLS}}$ and $\hat{\mu}_{\mathrm{OLS}}$ of the parameters $a$ and $\mu$, respectively, minimize the objective function

$$
Q_{n}^{\mathrm{OLS}}(a, \mu)=\sum_{i=1}^{n}\left(X_{i}-\mu a^{-(i-1)}\right)^{2} .
$$


It can be easily shown that $\hat{a}_{\mathrm{OLS}}$ is a solution to the equation

$$
\left(\sum_{i=1}^{n} \frac{X_{i}}{a^{i-1}}\right)\left(\sum_{i=1}^{n} \frac{i-1}{a^{2(i-1)}}\right)=\left(\sum_{i=1}^{n} \frac{(i-1) X_{i}}{a^{i-1}}\right)\left(\sum_{i=1}^{n} \frac{1}{a^{2(i-1)}}\right),
$$

and $\hat{\mu}_{\mathrm{OLS}}$ is given by

$$
\hat{\mu}_{\mathrm{OLS}}=\frac{\hat{a}_{\mathrm{OLS}}^{2}-1}{\hat{a}_{\mathrm{OLS}}\left(1-\hat{a}_{\mathrm{OLS}}^{-2 n}\right)} \sum_{i=1}^{n} \hat{a}_{\mathrm{OLS}}^{-i} X_{i} .
$$

3.2. The WLS method. In the model considered, the variances of the random variables $X_{i}, i=1, \ldots, n$, are not equal. They are of the form $\operatorname{Var}\left(X_{i}\right)=\sigma^{2} w_{i}$, where $w_{i}=a^{-2(i-1)}$ are unknown. Therefore instead of $Q_{n}^{\mathrm{OLS}}$, considered in [CYLH] , we propose taking as an objective function the following weighted sum of squares:

$$
Q_{n}^{\mathrm{WLS}}(a, \mu)=\sum_{i=1}^{n}\left(X_{i}-\mu a^{1-i}\right)^{2} \hat{a}^{2(i-1)},
$$

where $\hat{a}$ is a consistent estimator of $a$, for example $\hat{a}=\hat{a}_{\mathrm{LS}}$. The so-called weighted least squares (WLS) estimator $\hat{a}_{\mathrm{WLS}}$ of $a$ minimizes $Q_{n}^{\mathrm{WLS}}$.

3.3. The PWLS method. Treating $a$ as known, we can estimate $\mu=$ $E\left(X_{1}\right)=E\left(Y_{i}\right)$ based on the i.i.d. observations $Y_{i}=a^{i-1} X_{i}, i=1, \ldots, n$, by

$$
\hat{\mu}=\frac{1}{n} \sum_{i=1}^{n} a^{i-1} X_{i} .
$$

Then, replacing the unknown parameter $\mu$ in 3.1 by $\hat{\mu}$, we obtain the objective function

$$
Q_{n}^{\mathrm{PWLS}}(a)=\sum_{i=1}^{n}\left(X_{i}-\hat{\mu} a^{1-i}\right)^{2} \hat{a}^{2(i-1)} .
$$

The so-called profile weighted least squares (PWLS) estimator $\hat{a}_{\mathrm{PWLS}}$ of the parameter a minimizes $Q_{n}^{\mathrm{PWLS}}$.

REMARK 3.1. In a similar fashion one could introduce a profile-LS method. Replacing the unknown parameter $\lambda$ in $(2.3)$ by its consistent estimator

$$
\hat{\lambda}=\frac{1}{n} \sum_{i=1}^{n}\left[(i-1) \ln a+\ln X_{i}\right]
$$

yields an objective function that depends only on one parameter, $\vartheta=\ln a$. It is straightforward to verify that the solution of the corresponding optimization problem is given by (2.4). Therefore the profile-LS method and the LS method lead to the same estimator of $a$. 
4. ML estimators in some special cases. The estimators we have described so far are based on the semiparametric approach, since no assumptions on the cumulative distribution function $F$ have been made. In a simulation study we compare the accuracy of the proposed semiparametric estimators of $a$ with the known estimators given by Lam [L3] and Chan et al. CYLH], and also we examine how much we lose using the semiparametric estimates instead of the ML estimates when ones assumes a particular form of the distribution function $F$. The ML estimation of $a$ was studied by Lam and Chan $[\mathrm{LC}]$ for the lognormal distribution function $F$, by Aydoğdu et al. ASK] for the Weibull distribution, and by Chan et al. [CLL for the gamma distribution.

Notice that if $f(x)$ is the probability density function of the random variable $X_{1}$, then the probability density function of $X_{i}=a^{1-i} X_{1}, i=$ $2, \ldots, n$, is $a^{i-1} f\left(a^{i-1} x\right)$. Therefore the likelihood function $L$ based on the observations $x_{1}, \ldots, x_{n}$ of the geometric process $\left\{X_{i}: i=1, \ldots, n\right\}$ with unknown ratio parameter $a$ is

$$
L\left(a ; x_{1}, \ldots, x_{n}\right)=a^{n(n-1) / 2} \prod_{i=1}^{n} f\left(a^{i-1} x_{i}\right) .
$$

4.1. Lognormal distribution. If $X_{1}$ has the lognormal distribution with probability density function

$$
f(x ; \alpha, \beta)=\frac{1}{\sqrt{2 \pi} \beta x} \exp \left[-\frac{(\ln x-\alpha)^{2}}{2 \beta^{2}}\right] \mathbf{1}_{(0, \infty)}(x),
$$

then under the observations $x_{1}, \ldots, x_{n}$ of the geometric process with unknown ratio $a$, the likelihood function is

$$
\begin{aligned}
& L\left(a, \alpha, \beta ; x_{1}, \ldots, x_{n}\right) \\
& =(\beta \sqrt{2 \pi})^{-n}\left(\prod_{i=1}^{n} \frac{1}{x_{i}}\right) \exp \left\{-\frac{1}{2 \beta^{2}} \sum_{i=1}^{n}\left[(i-1) \ln a+\ln x_{i}-\alpha\right]^{2}\right\} .
\end{aligned}
$$

It is straightforward to verify that the ML estimator $\hat{a}_{\mathrm{ML}}$ of $a$ is given by (2.4) (see [LC] for more details). If $Y_{i}, i=1, \ldots, n$, are lognormally distributed then the errors $e_{i}, i=1, \ldots, n$, in model (2.1) are normally distributed with equal variances. It is well known fact that in this case LS estimators are identical to ML estimators.

4.2. Gamma distribution. For the geometric process with gamma distribution function $F$, i.e. when $X_{1}$ has the gamma distribution with probability density function

$$
f(x ; \alpha, \beta)=\frac{\beta^{\alpha}}{\Gamma(\alpha)} x^{\alpha-1} \exp (-\beta x) \mathbf{1}_{(0, \infty)}(x),
$$


the likelihood function is

$$
L\left(a, \alpha, \beta ; x_{i}, \ldots, x_{n}\right)=\frac{a^{n(n-1) \alpha / 2} \beta^{n \alpha}}{\Gamma(\alpha)^{n}} \exp \left(-\beta \sum_{i=1}^{n} a^{i-1} x_{i}\right) \prod_{i=1}^{n} x_{i}^{\alpha-1} .
$$

From the likelihood equations one can easily obtain a formula for the ratio parameter estimator. The ML estimator of the parameter $a$, denoted by $\hat{a}_{\mathrm{MLG}}$, is a solution to the equation

$$
\sum_{i=1}^{n}(n-2 i+1) a^{i-1} x_{i}=0 .
$$

The asymptotic properties of the estimator $\hat{a}_{\mathrm{MLG}}$, as well as of ML estimators of $\alpha$ and $\beta$, can be found in CLL.

4.3. Weibull distribution. For the geometric process with Weibull distribution function $F$, i.e. for the case when $X_{1}$ has the Weibull distribution with probability density function

$$
f(x ; \alpha, \beta)=\frac{\alpha}{\beta^{\alpha}} x^{\alpha-1} \exp \left[-\left(\frac{x}{\beta}\right)^{\alpha}\right] \mathbf{1}_{(0, \infty)}(x),
$$

the likelihood function is

$$
L\left(a, \alpha, \beta ; x_{1}, \ldots, x_{n}\right)=\frac{\alpha^{n} a^{(n-1) n \alpha / 2}}{\beta^{n \alpha}} \exp \left(-\beta^{-\alpha} \sum_{i=1}^{n} a^{(i-1) \alpha} x_{i}^{\alpha}\right) \prod_{i=1}^{n} x_{i}^{\alpha-1} .
$$

In this case, the system of the likelihood equations has the form

$$
\left\{\begin{array}{l}
\frac{n(n+1)}{2}-\sum_{i=1}^{n} i\left(\frac{a^{i-1} x_{i}}{\beta}\right)^{\alpha}=0, \\
\frac{n}{\alpha}-n \ln \beta+\frac{n(n-1)}{2} \ln a+\sum_{i=1}^{n} \ln x_{i}-\sum_{i=1}^{n}\left(\frac{a^{i-1} x_{i}}{\beta}\right)^{\alpha} \ln \left(\frac{a^{i-1} x_{i}}{\beta}\right)=0, \\
n-\sum_{i=1}^{n}\left(\frac{a^{i-1} x_{i}}{\beta}\right)^{\alpha}=0 .
\end{array}\right.
$$

The above system cannot be solved analytically, and numerical solutions are unstable since the equations involve all parameters through power functions (for details see CLL]). Therefore, in our simulation study, we used the methodology of modified maximum likelihood (MML) estimation introduced by Aydoğdu et al. ASK. This method gives explicit formulas for estimators of the ratio parameter $a$ and of the distribution parameters $\alpha$ and $\beta$. The basic idea behind the MML approach is to write the likelihood equations in terms of some ordered and normalized variates and to linearize the intractable exponent functions around their expected values. The algorithm for computing the MML estimators for Weibull distribution is complicated; for more details the reader is referred to the original paper [ASK]. The MML 
approach involves a two-step procedure. In the first step we use the LS estimator for the ratio to compute the above-mentioned normalized variates (equation (4.1) in [ASK]). In the second step the MML estimators are calculated according to formula (4.4) in [ASK]. We denote the MML estimator of the parameter $a$ by $\hat{a}_{\mathrm{MMLW}}$.

REMARK 4.1. Aydoğdu et al. ASK proved that, under some regularity conditions, the MML estimators are asymptotically normally distributed and asymptotically equivalent to the ML estimators.

5. A simulation study. In this section, we investigate the performance of the estimators of the ratio parameter by means of Monte Carlo simulations. All the computations were done in the statistical computing language $\mathrm{R}$.

Computing the estimators $\hat{a}_{\text {SLS }}$ and $\hat{a}_{\text {PWLS }}$ requires numerical minimization of the objective functions (2.7) and (3.2), respectively. The Nelder-Mead simplex method was employed to solve the first of these optimization problems, while the Brent algorithm was used for the latter. Multiple starting points were considered for both procedures to obtain reliable solutions. The Newton-Raphson method was utilized to solve equation (4.1) for the maximum likelihood estimators in the case of the gamma distribution.

The empirical mean square error (MSE) of the estimator, defined as the sum of the squared empirical bias and the empirical variance, was calculated for sample sizes $n=10,30,100$ based on $\lfloor 500000 / n\rfloor$ Monte Carlo simulations $(\lfloor m\rfloor$ denotes the greatest integer no greater than $m)$. We have considered various distributions of $X_{1}$ : lognormal $\mathcal{L N}(0,1)$, Weibull $\mathcal{W}(\alpha, 1)$, gamma $\mathcal{G}(\alpha, 1)$, where $\alpha=1,2,4$ is the shape parameter. Those distribution types were chosen due to their prevalence in reliability problems. The scale parameter was equal to 1 , since all estimators, except $\hat{a}_{\text {SLS }}$, are scale invariant. In real life problems, the trend is usually small, so the simulations were performed for $a=0.9$ and $a=1.1$. Calculations for other values of $a$ yielded similar results and are not presented for the sake of brevity. Preliminary simulations revealed that the OLS and WLS estimators are unreliable and occasionally greatly overestimate the true parameter, which may be attributed to the outliers. Hence these estimators are omitted in the further analysis. Results obtained for the second order least squares estimator $\hat{a}_{T}$, with identity weighting matrix, will also be omitted due to poor performance and lack of scale invariance.

In Table 1 the simulated MSE's $\times n^{2}$ are given. It is clear that the PWLS estimator outperforms the commonly used LS estimator in all cases considered, except for lognormal distribution when the LS estimator is equivalent to the maximum likelihood estimator. Moreover one can notice that in most 
Table 1. Simulated MSE $\times n^{2}$ for estimators of the ratio $a$

\begin{tabular}{|c|c|c|c|c|c|c|c|c|}
\hline Distribution & $a$ & $n$ & $\mathrm{LS}$ & PWLS & SLS & TM & MLG & MMLW \\
\hline $\mathcal{W}(1,1)$ & 0.9 & 10 & 1.6924 & 1.3058 & 2.3517 & 1.7768 & 1.2048 & 1.2213 \\
\hline \multirow[t]{5}{*}{$=\mathcal{G}(1,1)$} & & 30 & 0.5377 & 0.3643 & 0.4213 & 0.5552 & 0.3479 & 0.3491 \\
\hline & & 100 & 0.1648 & 0.1041 & 0.1161 & 0.1655 & 0.1017 & 0.1019 \\
\hline & 1.1 & 10 & 2.5449 & 1.9429 & 3.9490 & 2.6779 & 1.7930 & 1.8170 \\
\hline & & 30 & 0.7926 & 0.5416 & 0.6181 & 0.8166 & 0.5162 & 0.5189 \\
\hline & & 100 & 0.2335 & 0.1459 & 0.1639 & 0.2367 & 0.1427 & 0.1432 \\
\hline \multirow[t]{6}{*}{$\mathcal{W}(2,1)$} & 0.9 & 10 & 0.4092 & 0.3183 & 0.5408 & 0.4365 & 0.3091 & 0.2983 \\
\hline & & 30 & 0.1338 & 0.0932 & 0.1073 & 0.1374 & 0.0923 & 0.0857 \\
\hline & & 100 & 0.0400 & 0.0270 & 0.0282 & 0.0404 & 0.0269 & 0.0249 \\
\hline & 1.1 & 10 & 0.5996 & 0.4701 & 0.9392 & 0.6382 & 0.4579 & 0.4445 \\
\hline & & 30 & 0.2001 & 0.1387 & 0.1550 & 0.2058 & 0.1374 & 0.1277 \\
\hline & & 100 & 0.0578 & 0.0389 & 0.0415 & 0.0575 & 0.0387 & 0.0361 \\
\hline \multirow[t]{6}{*}{$\mathcal{W}(4,1)$} & 0.9 & 10 & 0.1011 & 0.0852 & 0.1366 & 0.1069 & 0.0843 & 0.0742 \\
\hline & & 30 & 0.0331 & 0.0262 & 0.0299 & 0.0342 & 0.0261 & 0.0215 \\
\hline & & 100 & 0.0102 & 0.0078 & 0.0075 & 0.0101 & 0.0078 & 0.0063 \\
\hline & 1.1 & 10 & 0.1520 & 0.1275 & 0.2686 & 0.1613 & 0.1262 & 0.1108 \\
\hline & & 30 & 0.0501 & 0.0396 & 0.0516 & 0.0510 & 0.0395 & 0.0326 \\
\hline & & 100 & 0.0146 & 0.0113 & 0.0114 & 0.0151 & 0.0113 & 0.0091 \\
\hline \multirow[t]{6}{*}{$\mathcal{G}(2,1)$} & 0.9 & 10 & 0.6476 & 0.5599 & 0.9572 & 0.7305 & 0.5425 & 0.5532 \\
\hline & & 30 & 0.2042 & 0.1692 & 0.2036 & 0.2268 & 0.1669 & 0.1719 \\
\hline & & 100 & 0.0630 & 0.0490 & 0.0522 & 0.0684 & 0.0488 & 0.0501 \\
\hline & 1.1 & 10 & 0.9659 & 0.8376 & 1.4821 & 1.0936 & 0.8124 & 0.8284 \\
\hline & & 30 & 0.3144 & 0.2552 & 0.2941 & 0.3474 & 0.2515 & 0.2577 \\
\hline & & 100 & 0.0931 & 0.0731 & 0.0776 & 0.1016 & 0.0727 & 0.0753 \\
\hline \multirow[t]{6}{*}{$\mathcal{G}(4,1)$} & 0.9 & 10 & 0.2828 & 0.2622 & 0.4988 & 0.3324 & 0.2599 & 0.2720 \\
\hline & & 30 & 0.0922 & 0.0826 & 0.1289 & 0.1054 & 0.0822 & 0.0882 \\
\hline & & 100 & 0.0277 & 0.0245 & 0.0264 & 0.0318 & 0.0245 & 0.0267 \\
\hline & 1.1 & 10 & 0.4197 & 0.3913 & 0.7101 & 0.4919 & 0.3874 & 0.4072 \\
\hline & & 30 & 0.1396 & 0.1255 & 0.1483 & 0.1606 & 0.1249 & 0.1337 \\
\hline & & 100 & 0.0408 & 0.0367 & 0.0384 & 0.0477 & 0.0366 & 0.0400 \\
\hline \multirow[t]{6}{*}{$\mathcal{L} \mathcal{N}(0,1)$} & 0.9 & 10 & 1.0041 & 1.2815 & 1.6994 & 1.2248 & 1.2781 & 1.2403 \\
\hline & & 30 & 0.3248 & 0.4858 & 0.3842 & 0.3893 & 0.4621 & 0.4388 \\
\hline & & 100 & 0.1008 & 0.1683 & 0.1040 & 0.1204 & 0.1605 & 0.1475 \\
\hline & 1.1 & 10 & 1.4984 & 1.9079 & 2.7245 & 1.8318 & 1.9008 & 1.8446 \\
\hline & & 30 & 0.4801 & 0.7409 & 0.5765 & 0.5746 & 0.6991 & 0.6617 \\
\hline & & 100 & 0.1481 & 0.2426 & 0.1540 & 0.1754 & 0.2296 & 0.2106 \\
\hline
\end{tabular}


cases the MSE's of the estimators $\hat{a}_{\text {PWLS }}$ are smaller than the MSE's of all other semiparametric estimates. For models considered in Table 1, except the one based on lognormal distribution, using the PWLS method gives on average an about $22 \%$ reduction of MSE's of the estimators in comparison to the LS and SLS methods, and close to $27 \%$ in comparison to the TM method.

For the smallest sample size, $n=10$, the SLS method always yields the greatest MSE value. In order to compute $\hat{a}_{\text {SLS }}$ one has to estimate the weighting matrix given by (2.13), which involves large inaccuracy in estimation of the third and fourth moments of the errors. However, when $n$ increases, the SLS estimators become more accurate (in terms of MSE) than the LS estimators for the Weibull and gamma distributions. Hence one could expect that in these cases the SLS method is asymptotically more efficient than the LS method. This observation is consistent with Theorem 4 in [WL] which states that the SLS estimator is asymptotically more efficient than the LS estimator if the third moment of the random errors is nonzero (the third moments of the Weibull and gamma distributions are nonzero). On the other hand, for the lognormal distribution the MSE of the LS estimator is smaller than the MSE of the SLS estimator. However, the differences are less pronounced and we may expect that in this case these two methods are asymptotically equivalent (the third moment of the lognormal distribution is zero).

Based on the presented data we find the PWLS estimator to be on average the most accurate among all the nonparametric estimators that were tested. In contrast to the LS method, calculation of PWLS estimators involved numerical solution of an optimization problem, but given the modern computational power, the extra cost of the PWLS method over the LS method is negligible.

In Table 1 the results for the parametric methods of estimation, based on the maximum likelihood concept, are also given for completeness. As expected, they yield the smallest MSE when the maximum likelihood estimator is consistent with a particular form of the distribution function $F$. However, for models considered in Table 1, the average decrease of MSE due to utilizing the ML estimators, MLG and MMLW, instead of PWLS is only $7 \%$. Moreover our simulation study shows that the MMLW estimator yields larger MSE than the nonparametric PWLS estimator when it is misused and employed to gamma distributed data, namely $X_{1} \sim \mathcal{G}(\alpha, 1)$ with $\alpha=2,4$.

6. Real data analysis. In this section, six of the previously discussed estimation methods will be used to study real data sets. In order to measure the goodness-of-fit and compare models with different estimates of the ratio, we define the mean square error (MSE) and the maximum percentage error 
(MPE) as

$$
\mathrm{MSE}=\frac{1}{n} \sum_{i=1}^{n}\left(X_{i}-\hat{X}_{i}\right)^{2} \quad \text { and } \quad \mathrm{MPE}=\max _{1 \leq i \leq n}\left|\left(T_{i}-\hat{T}_{i}\right) / T_{i}\right|,
$$

where $T_{i}, i=1, \ldots, n$, is the occurrence time of the $i$ th event determined by $T_{i}=\sum_{j=1}^{i} X_{i}$. The fitted values of data, $\hat{X}_{i}$ and $\hat{T}_{i}$, were evaluated as $\hat{X}_{i}=\hat{\mu} / \hat{a}^{i-1}, i=1, \ldots, n$, where $\hat{\mu}=n^{-1} \sum_{i=1}^{n}\left(\hat{a}^{i-1} X_{i}\right)$ is an estimate of $\mu=E\left(X_{1}\right)$, and $\hat{T}_{i}=\sum_{j=1}^{i} \hat{X}_{j}$. This approach is based entirely on the ratio estimate and can be utilized to any of the estimators considered, whether based directly on observations (Section 3) or on logarithms of observations (Section 2). The MSE informs us about the overall fitting, while the MPE concerns the worst fit in the data set.

Table 2. Estimation of the ratio parameter and goodness of fit for real data sets

\begin{tabular}{l|ccc|ccc}
\hline \multirow{2}{*}{ Estimator } & \multicolumn{2}{|c|}{ Coal-mining disasters data } & \multicolumn{3}{c}{ No. 3 data } \\
& $\hat{a}$ & MSE & MPE & $\hat{a}$ & MSE & MPE \\
\hline LS & 0.990913 & 81788.8 & 0.5031 & 1.041649 & 196179.5 & 0.4063 \\
PWLS & 0.990914 & 81790.4 & 0.5030 & 1.028099 & 240645.6 & 0.6155 \\
SLS & 0.990658 & 81554.1 & 0.5150 & 1.037871 & 207641.0 & 0.4793 \\
TM & 0.990745 & 81631.8 & 0.5110 & 1.049272 & 185207.1 & 0.2599 \\
MLG & 0.990914 & 81789.9 & 0.5031 & 1.035476 & 215623.1 & 0.5189 \\
MMLW & 0.990803 & 81685.4 & 0.5083 & 1.036772 & 211262.2 & 0.4980 \\
\hline
\end{tabular}

The first data set is known as "the coal-mining disasters data" and it has 190 observations of interval times in days between successive disasters in coal mines in Great Britain during over a century (see $[\mathrm{AH}]$ ). The data set contains one zero which indicates that there were two accidents the same day. This observation was adjusted to 0.5 because our estimation methods require inter-arrival times to be positive. Moreover it is reasonable to assume that probably those two accidents did not occur at exactly the same time. Estimates of the ratio $a, \mathrm{MSE}$ and MPE are given in Table 2. We can see that all the estimation methods yield very similar results. The SLS estimator is the best according to MSE, while the PWLS method gives the smallest MPE. The MSE of the MMLW and LS estimates are only $0.16 \%$ and $0.29 \%$ greater than the MSE of the SLS estimate, respectively.

The No. 3 data set contains 71 times in hours for the unscheduled maintenance actions for U.S.S. Halfbeak No. 3 propulsion diesel engine. It was originally studied in $\mathrm{AF}$, and then in [CLL, who showed the data are consistent with the GP model and that the ratio is significantly greater than 1 . Comparison of the models that employ different estimates is given in Table 2 . The trimean method, which has yielded the largest estimate for the ratio, 
gives the smallest mean square and maximum percentage errors, $5.6 \%$ and $36 \%$ reductions compared to the LS method, respectively. Both the TM and SLS estimators outperform the maximum likelihood estimators (MLG and MMLW) in terms of MSE and MPE.

It should be noted that the results presented for the LS and MMLW estimators are consistent with the results given in [ASK], likewise the results for the MLG estimators agree with those in CLL].

7. Conclusions and some prospects. In this paper we have proposed semiparametric estimators of the ratio of a geometric process. In the simulation studies we have compared their accuracy with the accuracy of the estimator proposed by Lam [L3] as well as with the accuracy of the ML estimators.

We have been mainly concerned with finite sample performance of the estimators considered. In the future we plan to examine the asymptotic properties of the estimators proposed. The estimators considered in Sections 2.1. 2.3 and 3.1 are minimum contrast estimators (MCE) (see e.g. [BS]) and their strong consistency can be deduced, under some assumptions on the unknown distribution function $F$, from strong consistency results for MCE's (see [BS]). Examining the strong consistency of the estimators considered in Sections 2.2 and 3.3 can be a much more difficult task.

Our simulation study revealed that the PWLS method, introduced in Section 3.3 provides very accurate and reliable semiparametric estimators of the geometric process ratio. In future work we intend to investigate the consistency and other asymptotic properties of PWLS estimators. As this method requires numerical optimization of a one-dimensional function and no explicit equations for the ratio parameter $a$ can be provided, the theoretical investigation will be based on the theory of estimating functions (see e.g. [G]).

The estimation methods proposed can also be applied to estimate the ratio parameter of an extended geometric process, i.e. a stochastic process $\left\{X_{i}\right.$, $i=1,2, \ldots\}$ for which there exists a positive real number $a$ and a nondecreasing sequence $\left\{b_{i}, i=1,2, \ldots\right\}$ such that $\left\{Y_{i}=a^{b_{i}} X_{i}, i=1,2, \ldots\right\}$ is a renewal process (see for example $[\mathrm{BM}]$ ).

Nonparametric estimation of the unknown cumulative distribution function $F$, the renewal distribution of the process $\left\{Y_{i}, i=1,2, \ldots\right\}$, is also an interesting problem which we plan to take up in the future.

\section{References}

[AH] D. F. Andrews and A. M. Herzberg, Data, Springer, New York, 1985.

[AF] H. Ascher and H. Feingold, Repairable Systems Reliability, Dekker, New York, 1984 . 
[ASK] H. Aydoğdu, B. Şenoğlu and M. Kara, Parameter estimation in geometric process with Weibull distribution, Appl. Math. Comput. 217 (2010), 2657-2665.

[BM] L. Bordes and S. Mercier, Extended geometric processes: Semiparametric estimation and applications to reliability, J. Iranian Statist. Soc. 12(1) (2013), $1-34$.

[BS] A. Bose and D. Sengupta, Strong consistency of minimum contrast estimators with applications, Sankhyā 65 (2003), 440-463.

[BLZ] W. J. Braun, W. Li and Y. Q. Zhao, Properties of the geometric and related processes, Naval Res. Logistics 52 (2005), 607-616.

[CLL] J. S. K. Chan, Y. Lam and D. Y. P. Leung, Statistical inference for geometric process with gamma distributions, Comput. Statist. Data Anal. 47 (2004), 565581.

[CYLH] J. S. K. Chan, P. L. H. Yu, Y. Lam and A. P. K. Ho, Modelling SARS data using treshold geometric process, Statist. Medicine 25 (2006), 1826-1839.

[C] Y. L. Chen, A bivariate optimal imperfect preventive maintenance policy for a used system with two-type shocks, Computers Industrial Engrg. 63 (2013), 1227-1234.

[F] M. S. Finkelstein, A scale model of general repair, Microelectronics and Reliability 33 (1993), 41-44.

[G] V. P. Godambe, The foundations of finite sample estimation in stochastic processes, Biometrika 419 (1985), 428-72.

[KZSA] M. Kamal, S. Zarrin, S. Saxena and Arif-Ul-Islam, Weibull geometric process model for the analysis of accelerated life testing with complete data, Int. J. Statist. Appl. 2 (2012), 60-66.

[KB] R. Koenker and G. Basset, Regression quantiles, Econometrica 46 (1978), 33-50.

[L1] Y. Lam, Geometric process and replacement problem, Acta Math. Appl. Sinica 4 (1988a), 366-377.

[L2] Y. Lam, A note on the optimal replacement problem, Adv. Appl. Probab. 20 (1988b), 479-482.

[L3] Y. Lam, Nonparametric inference for geometric processes, Comm. Statist. Theory Methods 21 (1992), 2083-2105.

[L4] Y. Lam, The Geometric Process and its Applications, World Sci., Singapore, 2007.

[LC] Y. Lam and S. K. Chan, Statistical inference for geometric processes with lognormal distribution, Comput. Statist. Data Anal. 27 (1998), 99-112.

[LZ] Y. Lam and Y. L. Zhang, Analysis of a two-component series system with a geometric process model, Naval Res. Logistics 43 (1996), 491-502.

[LZCL] Y. Lam, L. X. Zhu, J. S. K. Chan and Q. Liu, Analysis of data from a series of events by a geometric process model, Acta Math. Appl. Sinica 20 (2004), 263-282.

[S] J. Stanley, On geometric processes and repair replacement problems, Microelelectronics and Reliability 33 (1993), 489-491.

[T] M. L. Tiku, Estimating the mean and standard deviation from censored normal samples, Biometrika 54 (1967), 155-165.

[WZ] G. J. Wang and Y. L. Zhang, Geometric process model for a system with inspections and preventive repair, Computers Industrial Engrg. 75 (2014), 13-19.

[WP] H. Wang and H. Pham, Reliability and Optimal Maintenance, Springer, 2006.

[W1] L. Wang, Estimation of nonlinear Berkson-type measurement error models, Statist. Sinica 13 (2003), 1201-1210.

[W2] L. Wang, Estimation of nonlinear models with Berkson measurement errors, Ann. Statist. Medicine 32 (2004), 2559-2579. 
[WL] L. Wang and A. Leblanc, Second-order nonlinear least squares estimation, Ann. Inst. Statist. Math. 60 (2008), 883-900.

[YTF] M. M. Yu, Y. H. Tang and Y. H. Fu, A geometric process model for $M / P H$ $(M / P H) / 1 / K$ queue with new service machine procurement lead time, Int. J. Systems Science 44 (2013), 1061-1075.

[YTLC] M. M. Yu, Y. H. Tang, L. Liu and J. Cheng, A phase-type geometric process repair model with spare device procurement and repairman's multiple vacations, Eur. J. Operational Res. 225 (2013), 310-323.

Alicja Jokiel-Rokita, Rafał Topolnicki

Faculty of Pure and Applied Mathematics

Wrocław University of Science and Technology

Wybrzeże Wyspiańskiego 27

50-370 Wrocław, Poland

E-mail: alicja.jokiel-rokita@pwr.edu.pl rafal.topolnicki@pwr.edu.pl 
